\title{
V: EDITIONSGRUNDSÄTZE
}

Leithandschrift ist der Frankfurter Kodex Fr, der jeweils nur auf der linken Seite abgedruckt wird. Um der Textstrukturierung willen sind die einzelnen Perikopen bzw. Perikopenverweise durchnumeriert und die Bibelstelle hinzugefügt. Der textkritische Apparat erscheint jeweils unter dem Perikopenblock. Auf den rechten Seiten stehen unter der entsprechenden Perikopennummer die Siglen der übrigen Textzeugen in der Reihenfolge Br N1 N2, deren Perikopenüberschriften und ihr auf Fr bezogener textkritischer Apparat. Wenn die Leithandschrift ausfallt, wird auf der rechten Seite die entsprechende Perikope nach der Bremer bzw. einer der beiden Nümberger Handschriften kursiv abgedruckt. Diese Verteilung auf die geraden bzw. ungeraden Seiten wird allerdings bei den vier Passionen durchbrochen, die nur in N2 (Perikopen 84, 86, 87) bzw. in Br und N2 (Perikope 90) überliefert sind. Andernfalls würden sich zu viele Leerseiten ergeben. Durch entsprechende Hinweise und vor allem durch den Kursivdruck ist einer möglichen Verwechslung mit dem Text der Leithandschrift vorgebeugt. Bei der nur in Fr überlieferten Passionsharmonie nach dem 'Leben Jhesu'1 (Perikope 89) werden jeweils auf der rechten Seite die entsprechenden Passagen aus der Berliner Handschrift (Sigle B) in kleinerer Kursivschrift wiedergegeben. Im Apparat erscheinen zusätzlich die Lesarten der Hamburger Handschrift (Sigle U). In Fr sind hier die Bibelstellen in runden bzw. in geschweiften Klammern - dies bei den in der Edition des 'Leben Jhesu' nicht vorkommenden Stellen - dem Text eingefügt; sie sind also nicht in der Handschrift überliefert. Diese Textanordnung insgesamt wurde vor allem gewählt, um der bis heute noch nicht aufgearbeiteten Quellenlage im Bereich der spätmittelalterlichen Plenarien ${ }^{2}$ Rechnung zu tragen. Sollte sich aufgrund späterer Handschriftenfunde die Überlieferungslage grundsätzlich ändern, so wäre zumindest der Editionsteil der Frankfurter Evangelienhandschrift nicht betroffen.

1 Vgl. den Exkurs S. LIIIff.

2 Dazu vgl. H. ReINITZER, 'Plenarien'. In: VL, 2. Auflage, Bd. 7, 1989, Sp. $738 f$. 
Die Leithandschrift wird grundsātzlich originalgetreu abgedruckt, ebenso die an ihre Stelle tretenden übrigen Handschriften. Die Abbreviaturen werden ohne Kennzeichnung aufgelöst und nur in Zweifelsfallen im Apparat verzeichnet. Die folgende Auflistung gibt die im volkssprachigen Text von Fr überlieferten Abbreviaturen mit ihren Auflösungen:

$\overline{\mathrm{a}}=\mathrm{an}, \mathrm{am} / \overline{\mathrm{e}}=\mathrm{en}, \mathrm{em} / \overline{\mathrm{i}}=\mathrm{in}, \mathrm{im} / \overline{\mathrm{m}}=\mathrm{mm} / \overline{\mathrm{n}}=\mathrm{nn} / \overline{\mathrm{o}}=$ on $/ \overline{\mathrm{p}}=$ pre $/ \overline{\mathrm{u}}=$ un, $\mathrm{um} / \overline{\mathbf{v}}=\mathrm{vm} / \overline{\mathbf{y}}=\mathrm{yn}, \mathrm{ym}$.

a übergeschrieben $=\mathrm{ra}$

$$
=\text { ar in wart }
$$

$\mathrm{e}^{\mathrm{w}}=$ ewangelium

$\overline{\mathrm{m}}=$ men

in mensche und den entsprechenden Wortformen sowie in der Ableitung menscheit

q mit übergeschriebenem $a=$ qua in quam

uñ = und (nur zweiter Schreiber)

vñ $=$ vnde (erster Schreiber), vnd (zweiter Schreiber)

$\mathrm{z}$ am Wortende = et in gerumet

Der übergeschriebene bzw. angehängte $e r$-Haken steht auch für einfaches $r$, in grozer für ro. Ebenfalls mit einem Haken wird $i r$ in wirdet bzw. wirdit, wirdig und wir gekürzt.

Dagegen kommen die folgenden Abbreviaturen nur in den lateinischen Textpassagen und in den Namen vor:

bz am Wortende $=$ bus $/ 1$ am Wortende mit Abkürzungshaken $=$ lis $/$ oR am Wortende mit Querstrich durch den verlängerten diagonalen Abstrich des R = orum / p mit geradem Querstrich durch die Unterlänge $=$ par $/ \mathrm{p}$ mit übergeschriebenem $\mathrm{i}=$ pri $/ 9$ am Wortende $=$ us

c mit Abkürzungshaken $=$ cetera $/ \mathrm{dn̄i}, \mathrm{dn̄j}=$ domini $/$ noīe $=$ nomine $/$ plma mit Abkürzungshaken am $1=$ plurima $/ \overline{\mathrm{pr}}=$ pater $/ \overline{\mathrm{pt}}=$ potest $/ \mathrm{s}$ mit Abkürzungshaken, scdm mit Abkürzungshaken am $d=\operatorname{secundum~} / \mathrm{B}=\operatorname{sed} /$ tp̄e, tempe jeweils mit geradem Querstrich durch die Unterlänge $=$ tempore $/$ ul mit schrägem Strich durch das $1=$ uel $/$ ur̃m = uestrum

ihm mit Abkürzungshaken am $\mathrm{h}=$ ihesum

ihs, ihus jeweils mit Abkürzungshaken am $h=$ ihesus

ihu mit Abkürzungshaken am $\mathbf{h}=$ ihesu

iohes mit Abkürzungshaken $\mathrm{am} \mathrm{h}=$ iohannes 
iohis mit Abkürzungshaken am $\mathrm{h}=$ iohannis

jhm mit Abkürzungshaken am $\mathrm{h}=$ jhesum

jhs, jhus jeweils mit Abkürzungshaken am $\mathrm{h}=$ jhesus

johem mit Abkürzungshaken am $h=$ johannem

johes mit Abkürzungshaken am $\mathrm{h}=$ johannes

johis mit Abkürzungshaken am $\mathrm{h}=$ johannis

math $^{\mathrm{m}}$, math $\mathrm{e}^{\mathrm{m}}=$ matheum

In den drei übrigen Handschriften begegnen außerdem noch folgende Abbreviaturen im volkssprachigen Text:

...ch am Wortende $=\ldots$ chen $/$ er-Haken $=\mathrm{er}, \mathrm{re} / \mathrm{i}$ hakenförmig, übergeschrieben $=\mathrm{r}$, ir $/ \mathrm{t}=\mathrm{el} / \overline{\mathrm{n}}=\mathrm{en} / \mathrm{o}$ übergeschrieben $=$ ro $/ \overline{\mathrm{o}}=\mathrm{om} / \mathrm{p}$ mit geschwungenem Strich durch die Unterlänge $=$ pro $/ \overline{\mathrm{r}}$ am Wortende $=\mathrm{m} / \mathrm{s}$ mit hakenförmigem Strich durch den Schaft $=\operatorname{ser} / \mathrm{u}$ übergeschrieben $=\mathrm{ur}, \mathrm{ru} / \overline{\mathrm{u}}=\mathrm{ün} / \overline{\mathrm{v}}=\mathrm{vn}$

ew, ewan jeweils mit abschließender Schleife; ewange ${ }^{m}$, ewangeli ${ }^{m}$, ew $\mathbf{w}^{\mathbf{m}}=$ ewangelium geschr mit abschließender Schleife $=$ geschriben $/$ getwer $=$ getruwer $/$ gewandg = gewandes / offent = offenlich / pphe mit geschwungenem Querstrich durch die Unterlängen der ligierten $\mathrm{pp}=$ prophete $/ \mathrm{pph}$ en mit geschwungenem Querstrich durch die Unterlängen der ligierten $\mathrm{pp}=$ propheten $/ \mathrm{sfe}=$ sancte $/ \mathrm{vorbz}=\mathrm{vorbaz} / \mathrm{w} \overline{\mathrm{n}}=\mathrm{wnd} / \mathrm{wz}=\mathrm{waz} / \mathrm{Win}-$ kelhaken $=$ vnd

Nur in lateinischen Textpassagen und in Namen ${ }^{3}$ begegnen in den drei übrigen Handschriften noch folgende Abbreviaturen:

$\mathrm{b}=\mathrm{bis} / \mathrm{t}=\mathrm{ul} / \mathrm{t}$ am Wortende mit abschließender Schleife $=\mathrm{tis} / \mathrm{t}^{\prime}=\mathrm{tur} / \mathrm{z}$ geschweift, am Wortende $=\mathrm{m} /$ spiegelverkehrtes $\mathrm{c}=$ con aūt $=$ autem $/$ ciui $^{\text {te }}=$ ciuitate $/ \mathrm{d}^{\prime}=\mathrm{de} /$ dice mit Abkürzungshaken $=\mathrm{dicetur} / \mathrm{dix} \mathrm{t}^{\mathrm{t}}=$ dixit $/ \overline{\mathrm{e}}=$ est $/$ eêt $=$ esset $/ \mathrm{ffe}=$ facte $/ \mathrm{ftus}=$ factus $/$ homis $=$ hominis $/$ hüt $=$ habuit $/$ if $=$ item $/ \mathrm{jE}=\mathrm{jtem} / \mathrm{pcto}=$ peccato $/$ postq̊ mit geschwungenem Strich durch die Unterlänge von $q=$ postquam / pris $=$ patris $/ \mathrm{q}$ mit geschwungenem Abstrich durch den Schaft $=$ quod $/ \mathrm{qz}=$ quia $/$ qdam $=$ quidam $/ \mathrm{s}$. = secundum $/ \mathrm{sabbi}=$ sabbati $/ \mathrm{spüs}=$ spiritus / stus $=$ sanctus $/ B=\operatorname{sed} / B^{m}=$ secundum $/$ tpe mit und ohne geradem Querstrich durch die Unterlänge $=$ tempore $/ \overline{\mathrm{vm}}=$ verbum $/ \mathrm{vīm}=$ vestrum $/ \mathrm{zt}$ mit abschließender

3 Groß- bzw. Kleinschreibung werden hier in der Auflistung nicht eigens unterschieden. 


\section{LXXVI}

Schlaufe $=$ zetera $/$ Winkelhaken $=$ et $/$ et cetera wird durch eine der gängigen Siglen ${ }^{4}$ wiedergegeben. Nur durch den Anfangsbuchstaben und einen Punkt sind mitunter einige oder alle Wörter des Satzes dixit ihesus discipulis suis abgekürzt.

chananem $\mathrm{e}^{\mathrm{m}}=$ chananeum

ihrlm ${ }^{5}$ mit Abkürzungsstrich $=$ iherusalem

ihes mit Abkürzungsstrich $=$ iohannes $^{6}$

ihum mit Abkürzungsstrich = ihesum

iohem, iohm jeweils mit Abkürzungsstrich = iohannem

iohns mit Abkürzungsstrich $=$ iohannes

jersalm mit Abkürzungsstrich $=$ jerusalem

jhoe mit Abkürzungsstrich $=$ johanne $^{7}$

jhnem mit Abkürzungsstrich $=$ johannem

jhrlm mit Abkürzungsstrich $=$ jherusalem

jhu mit Abkürzungsstrich $=$ jhesu

joh, johaes, johanns, johns, johs jeweils mit Abkürzungsstrich $=$ johannes

johais, johis jeweils mit Abkürzungsstrich $=$ johannis

johem, johm jeweils mit Abkürzungsstrich $=$ johannem

jrlm mit Abkürzungsstrich $=$ jerusalem

jrlimis mit Abkürzungsstrich $=$ jerualimis

luc mit doppeltem Abkürzungsstrich = lucas

magd $=$ magdalena $/$ magd $^{\circ}=$ magdalene

math, mth jeweils mit Abkürzungsstrich = matheus

mathm; mthm mit Abkürzungsstrich = matheum

$\mathbf{x} \bar{p} \mathbf{i}=$ christi $/ \mathbf{x} \overline{\mathbf{p} m}=$ christum $/ \mathbf{x} \bar{p} s, \mathbf{x} \bar{p} u s=$ christus

Groß- und Kleinschreibung der Handschriften werden jeweils beibehalten. Da vielfach nicht sicher zu entscheiden ist, ob Zusammen- oder Getrenntschreibung vorliegt, die Handschriften zudem in diesem Punkte nicht konsequent verfahren, sind die Komposita und Affixoide auch entgegen dem handschriftlichen Befund zusammengeschrieben. Die unterschiedlichen

4 Vgl. PAUl ARNOLD GruN, Schlüssel zu alten und neuen Abkürzungen, Limburg/Lahn 1966, S.298. Das zwanzigste Zeichen für et cetera in der ersten Kolumne entspricht in etwa dem hier gebrauchten.

5 In ihlrm mit Abkürzungsstrich - N1, fol.12r - sind $\mathrm{r}$ und 1 fillschlich vertauscht.

6 Durch EinfluB der Abbreviaturen für ihesus ist die in N2, fol.6r, überlieferte Abkürzung wohl aus iohes verschrieben.

7 In jhoe mit Abkürzungsstrich - N2, fol.114r - sind o und h falschlich vertauscht. 
$s$-Graphien sind zu $s$ vereinheitlicht. Die Interpunktion der Handschriften wird beibehalten, der Punkt aber grundsătzlich auf die Grundlinie gestellt, zumal eine klare Entscheidung über seine Position vielfach nicht möglich ist.

Fehlschreibungen werden durch Kursivdruck im Text hervorgehoben, bei kursivem Text dagegen durch gerade Schrift. Bei notwendigem Wortersatz bzw. bei sinnstörenden Auslassungen wird nach Möglichkeit ohne Anpassung an die Sprachform der Leithandschrift auf die anderen Handschriften zurückgegriffen. Notwendige Ergănzungen durch den Editor werden in eckige Klammern eingeschlossen und im Text durch Wechsel der Schriftart - kursiv bei gerader, gerade bei kursiver Schrift - gekennzeichnet. Nichtbiblische Passagen im Perikopentext ${ }^{8}$ sind durch ausgeblendete Schrift hervorgehoben. Die Zeilen- und Seitenenden werden durch eingefügte / bzw. // angezeigt. Die Paginierung ist am linken Rand vermerkt, an dem auch die für den Apparat erforderlichen Zeilenzahlen in Fünferschritten stehen. Die Paginierung der übrigen Textzeugen steht jeweils vor den auf der gegenüberliegenden Seite abgedruckten Perikopenüberschriften.

Im textkritischen Apparat unter dem Text werden wie üblich die Eingriffe in den Text vermerkt. Bei Abweichungen der Übersetzung vom Bibeltext und bei nicht sinnstörenden Auslassungen wird die entsprechende Vulgatastelle angeführt. Mit + wird ein Zusatz, mit - ein Fehlen und mit eine Umstellung gekennzeichnet. Bei mehreren gleichen Wortformen in einer Zeile wird durch Hochzahlen eine eindeutige Zuordnung sichergestellt. Der zweite Apparat ist verhältnismäßig ausführlich. Allerdings werden rein orthographische und und phonologische Varianten nicht berücksichtigt. Dasselbe gilt für sogenannte iterierende Varianten auf der Wortebene wie etwa da / do, das / diz oder (en ...) nicht / (en ...) nit. Auf morphologischer Ebene sind nur gravierende Abweichungen vermerkt, nicht aber beispielsweise solche Präfix- und Suffixvarianten wie zur- / zu- oder -nusze / -nisße. Die vielfach variierenden Namensformen des Textes werden nicht im Apparat, sondern in der separaten Namenliste 9 aufgeführt. Tritt an die Stelle der Leithandschrift eine der anderen Textzeugen, so gibt es nur einen kritischen Apparat.

8 Vgl. oben S. LXVIff.

9 Vgl. unten S. 374-387. 